\title{
Intravenous Methylprednisolone Pulse Therapy Versus Intravenous Immunoglobulin (IVIG) In The Prevention Of Coronary Artery Disease In Children With Kawasaki Disease: A Randomized Controlled Trial
}

Nahid Aslani

Isfahan University of Medical Sciences

Sayed-Reza Raeeskarami

Tehran University of Medical Sciences

Ehsan Aghaei-Moghadam

Tehran University of Medical Sciences

Fatemeh Tahghighi

Tehran University of Medical Sciences

Raheleh Assari

Tehran University of Medical Sciences

Peyman Sadeghi

Tehran University of Medical Sciences

Vahid Ziaee ( $\boldsymbol{D}$ ziaee@tums.ac.ir)

Tehran University of Medical Sciences https://orcid.org/0000-0003-4648-3573

\section{Research article}

Keywords: Kawasaki Disease, Coronary Artery Lesion, Intravenous Methylprednisolone Pulse, Intravenous Immunoglobulin, Steroid Therapy

Posted Date: May 6th, 2021

DOI: https://doi.org/10.21203/rs.3.rs-460428/v1

License: (9) This work is licensed under a Creative Commons Attribution 4.0 International License. Read Full License 


\section{Abstract}

\section{Background}

Kawasaki disease (KD) is often complicated by coronary artery damage, including dilatation or aneurysms. IVIG is used with aspirin to prevent coronary artery abnormalities in KD. Since the primary treatment for other vasculitis is the use of corticosteroids, this study has been performed to evaluate the effect of intravenous pulse methylprednisolone therapy in preventing coronary artery lesions in KD.

\section{Method}

A randomized, single-blind clinical trial was conducted on $40 \mathrm{KD}$ patients aged 6 months to 5 years. Patients were randomized into two groups according to the main treatment plan in addition to aspirin: case group (intravenous methylprednisolone pulse for three consecutive days and then oral prednisolone for 5 days) and control group (intravenous immunoglobulin 2 gram $/ \mathrm{kg}$ ). Echocardiography was performed for all children at least 3 times, during the acute phase, two weeks, and two months later.

\section{Results}

Data analysis at the end of the study was done on 40 patients (20 patients in each group). There were no significant differences in age and sex distribution, mean fever, and acute phase duration, as well as baseline echocardiography in 2 groups. The frequency of coronary artery lesion was $20 \%$ in the case group and $45 \%$ in the control group, after two weeks $(p<0.05)$, but there was no significant difference between 2 groups in types of coronary artery lesion after two weeks and the frequency and severity of coronary artery lesion after two months.

\section{Conclusion}

Intravenous methylprednisolone pulse as initial line therapy effectively control systemic and vascular inflammation and decrease coronary artery damage in KD.

\section{Background}

$\mathrm{KD}$ is an acute, self-limited medium vessel vasculitis most commonly affecting infants and children $<5$ years of age $(1,2)$. Complete KD requires persistent fever $\geq 5$ days plus 4 out of 5 clinical criteria, including bilateral nonexudative bulbar conjunctivitis, polymorphous non-vesicular rash, oropharyngeal changes, unilateral cervical lymphadenopathy, and swelling of extremities followed by desquamation (2). Patients who have a prolonged fever and show less than four clinical criteria have incomplete KD, and the diagnosis is based on laboratory findings or echocardiography.

Coronary artery aneurysms are a well-recognized complication of KD, occurring in roughly $20 \%$ of untreated diseases, and are the leading cause of acquired heart disease in developed countries $(3,4)$. Two classification criteria exist for the diagnosis of coronary artery dilation and aneurysms. The 
Japanese Ministry of Health (JHM) criteria classify coronary arteries using absolute or relative internal lumen diameter (5). Dilation is defined as an internal lumen diameter $>3 \mathrm{~mm}$ in children $<5$ years old or > $4 \mathrm{~mm}$ in children $\geq 5$ years old or if the internal diameter of a segment measures $\geq 1.5$ times that of an adjacent segment. JMH criteria are more commonly used in Japan, and given the variability in lumen sizes with respect to body size, it may underestimate the incidence of coronary artery dilations and aneurysms. The 2004 American Heart Association (AHA) adjusts for body surface area and classifies solely on Z-scores. Per AHA criteria, dilation is defined as a Z-score $\geq 2$ and $<2.5$, and aneurysms are diagnosed if $Z$-score is $\geq 2.5$. Dilation often resolves within $4-8$ weeks after fever onset. Giant aneurysms, defined as $\geq 8 \mathrm{~mm}$ per JHM and AHA or Z-score $\geq 10$ per AHA criteria, are unlikely to regress (6).

Furusho et al. first reported in 1983 that IVIG administered in the acute stage of KD rapidly eliminates inflammation symptoms and decreases the frequency of coronary artery lesions (CAL) to approximately $5 \%(7,8)$. Nearly 35 years later, according to the latest guidelines of the AHA in 2017, a single high dose of IVIG together with acetylsalicylic acid was still the standard recommended treatment for KD (6).

IVIG, also known as gamma globulin, is a therapeutic preparation comprising pooled blood donated from large numbers of healthy people. Although many clinical trials have demonstrated that immunoglobulin is effective and well-tolerated, various adverse effects have been reported. The majority of these events, such as flushing, headache, malaise, fever, chills, fatigue, and lethargy, are transient and mild. However, some rare side effects, including renal impairment, thrombosis, arrhythmia, aseptic meningitis, hemolytic anemia, and transfusion-related acute lung injury (TRALI), are severe. These adverse effects are associated with specific immunoglobulin preparations and individual differences (8). Approximately 5$35 \%$ of patients fail to respond to IVIG and remain febrile $\geq 36 \mathrm{~h}$ following completion of the IVIG infusion and are thus classified as IVIG-refractory or IVIG-resistant (9).

Corticosteroids are an effective treatment due to their well-known and strong anti-inflammatory properties applied in other forms of vasculitis. Their potential use in KD was considered soon after the disease was recognized and expected to benefit. Corticosteroids normalize multiple transcriptional patterns and inhibit the synthesis of the most known type 2 and proinflammatory cytokines (10). According to the AHA, intravenous methylprednisolone (IVMP), $30 \mathrm{mg} / \mathrm{kg}$ for three days with or without an oral glucocorticoid taper, is one of the three most second-line therapies 2017 Scientific Statement recommendation for these patients (6).

Potential benefits of corticosteroids as rescue therapy in $\mathrm{KD}$ also have been reported. Corticosteroids in $\mathrm{KD}$ have been studied both as primary therapy and "rescue" therapy, and doses have ranged from pulse doses of $30 \mathrm{mg} / \mathrm{kg}$ (maximum of $1 \mathrm{~g}$ ) to conventional anti-inflammatory doses ( $2 \mathrm{mg} / \mathrm{kg} / \mathrm{day}$ ). However, a few clinical studies have been conducted to evaluate the role of corticosteroids alone compared to IVIG. There have been many disagreements on the treatment, and none of the therapies tried thus far have proven to be sufficiently effective (11). This report deals with intravenous steroid pulse therapy to prevent 
aneurysm formation of the coronary arteries in the acute phase of KD by evaluating its effects using twodimensional echocardiography.

\section{Materials And Methods}

A randomized, single-blind clinical trial, registered in the Iranian Registry of Clinical Trials

(ClinicalTrials.IRCT Identifier: IRCT20181202041817N1), was performed from December 2019 until April 2020 in the pediatric rheumatology division of Children's Medical Center, a pediatric referral hospital, Tehran, Iran. The Ethics committee of the university approved the study. Consent forms were taken from patients' parents. The sample size was calculated by using the Altman nomogram with a power of $80 \%$. All the patients were followed up by phone.

The inclusion criteria were age group between 6 months to 5 years, symptoms in favor of complete and incomplete KD according to the AHA guideline of 2017 (6). Children with atypical KD, macrophage activation syndrome, recurrent or previous history of KD, previous confirmed coronary artery lesion, congestive heart failure, chronic kidney disease, sensitivity to methylprednisolone or prednisolone, active zoster virus infection or exposure to varicella for the last 21 days, intravenous or intramuscular usage of corticosteroids for more than three days in the last seven days, history of severe reactions to any human globulin product and registration in another study were excluded from the study.

The diagnosis of KD was based on the AHA criteria for complete and incomplete KD (6). Patients were observed over two months and underwent two-dimensional echocardiography periodically. The first echocardiography was performed at the diagnosis and on the first day of hospitalization, while the second and third echocardiography was done after two weeks and two months. A pediatric cardiologist evaluated the echocardiography for all patients who were completely blinded to the method and treatment outcome by the Samsung HS70 device 8-12 and 4-8 probes sizes. Coronary artery dimension average was measured in the short-axis view and diastolic phase of at least three consecutive cardiac cycles. Echocardiographic views of coronary arteries included RCA, LMCA, LCX proximal segment, and LAD proximal middle segment. Subsequent echocardiography was performed at least two more times, two weeks and two months later, based on coronary artery involvement and the cardiologist's discretion.

The coronary artery lesions were classified further as ectasia (diffuse dilatation), small aneurysm (local dilatation of internal lumen diameter less than $4 \mathrm{~mm}$ or $\geq 1.5$ times to the adjacent segment in children older than five years), medium aneurysm (local dilatation of internal lumen diameter $>4 \mathrm{~mm}$ but less than $8 \mathrm{~mm}$ or internal lumen diameter more than 1.5 to 4 times to the adjacent segment in children older than 5 years), and large or giant aneurysm (local dilatation of internal lumen diameter $>8 \mathrm{~mm}$ or $>4$ times to the adjacent segment in children older than five years) based on the Japanese Ministry of Health (JHM) criteria (5).

Patients who met the inclusion criteria according to the main treatment in addition to aspirin were randomly allocated into either case group (intravenous methylprednisolone pulse, $30 \mathrm{mg} / \mathrm{kg} / \mathrm{day}$ for three consecutive days and then oral prednisolone $1 \mathrm{mg} / \mathrm{kg} /$ day for three days group) and control group 
(intravenous immunoglobulin $2 \mathrm{gram} / \mathrm{kg}$ ) in a single-blinded approach, CONSORT flow diagram (Fig. 1). Once the patient's parents signed the informed consent, the physician administered an intravenous methylprednisolone pulse to each new patient based on the randomization protocol. The definition of response to treatment was based on the cessation of fever after 12 hours of IVIG infusion or the first dose of steroid pulse and reduced CRP to less than half before starting treatment. Patients who did not meet the treatment response criteria were considered treatment failure and excluded from the study, and received standard treatment (second dose of IVIG $2 \mathrm{~g} / \mathrm{kg}$ in the control group and IVIG $2 \mathrm{~g} / \mathrm{kg}$ in the case group).

Demographic, clinical, and laboratory findings of the participants were recorded, including age, sex, ESR, CRP, CBC with diff, SGOT, SGPT, urinalysis, urine culture, echocardiography results, and early-onset complications.

Data were analyzed using the SPSS for Windows (SPSS Inc., Chicago, IL) version 24 software. The Kolmogorov-Smirnov test checked the normality of variables. Mean (SD) and median (interquartile range) were used for expressing normally and non-normally distributed continuous variables. Categorical variables were reported as percentages. Mean (SD) and median (interquartile range) of continuous variables were compared between 2 groups using an independent t-test. For evaluation of the prevalence between 2 groups, Chi-square test and for evaluation of percentage McNemar test was applied. P-values less than 0.05 were considered significant.

\section{Results}

We recruited 41 participants in the study: 21 children in the control group and 20 children in the case group. One child in the control group refused to perform the second echocardiography. All of the patients met the treatment response criteria, and none of them were not considered treatment failures, and no participants were excluded from the study. Finally, data analysis for the findings, which was performed at the end of the study, was done on 40 patients (20 patients in the case group and 20 patients in the control group).

Patient demographic, clinical, and laboratory characteristics were similar between groups, with few exceptions. Table 1 showed the age and sex distribution of patients in both groups. There was no significant difference between the two groups. 
Table 1

Age and sex distribution of patients with Kawasaki disease

\begin{tabular}{|llll|}
\hline Sex/age & IVIG group & IVMP group & Total number (\%) \\
\hline Female & $9(45)$ & $13(65)$ & $22(55)$ \\
\hline Male & $11(5)$ & $7(35)$ & $18(45)$ \\
Mean Age & $2.7 y r$ & $2.6 y r$ & $40(100)$ \\
\hline \multicolumn{2}{|l}{ IVIG: Intravenous Immunoglobulin; IVMP: Intra Venous } & Methylprednisolone Pulse \\
\hline
\end{tabular}

The mean intervals from the onset of the fever to the start of treatment were 8.9 days in the case to 9.7 days in the control groups and were not significantly different $(P=0.3)$.

Also, the mean duration of the acute phase from the start of treatment to the reduction CRP index to more than $50 \%$ of initial values was about 7 hours and not significantly different between the two groups $(P=$ 0.9). No significant difference between groups also was seen in the complete and incomplete KD ratio. (Table 2).

Table 2: clinical type, mean fever and acute phase duration distribution of patients with Kawasaki disease

\begin{tabular}{|c|c|c|c|c|}
\hline Variables & IVIG group & IVMP group & Total number (\%) & P. value \\
\hline Complete Kawasaki disease & $8(40)$ & $13(65)$ & $21(52.5)$ & \multirow[t]{2}{*}{0.1} \\
\hline Incomplete Kawasaki disease & $12(60)$ & $7(35)$ & $19(47.5)$ & \\
\hline Mean fever duration & 9.7 days & 8.9days & $40(100)$ & 0.3 \\
\hline Mean acute phase of disease & 6.9 hours & 7 hours & $40(100)$ & 0.9 \\
\hline
\end{tabular}

IVIG: Intravenous Immunoglobulin; IVMP: Intra Venous Methylprednisolone Pulse

The summary of echocardiographic findings has been shown in Table 3. The baseline echocardiography did not significantly differ in 2 groups; coronary artery ectasia was the most common type of coronary involvement in both groups, and there was only one patient with a large coronary artery aneurysm in the control group. 
Table 3

Assessment of lesions of the first and second and third echocardiography

\begin{tabular}{|c|c|c|c|}
\hline Echocardiography & $\begin{array}{l}\text { IVIG group } \\
\text { Total number(\%) }\end{array}$ & $\begin{array}{l}\text { IVMP group } \\
\text { Total number(\%) }\end{array}$ & P. value \\
\hline \multicolumn{3}{|c|}{ First Echocardiography } & \multirow[t]{5}{*}{0.2} \\
\hline Ectasia & $14(70)$ & $9(45)$ & \\
\hline Small Aneurysm & $O(0)$ & $O(0)$ & \\
\hline Moderate Aneurysm & $O(0)$ & $O(0)$ & \\
\hline Giant Aneurysm & $1(5)$ & $O(0)$ & \\
\hline \multicolumn{3}{|c|}{ Second Echocardiography } & \multirow[t]{5}{*}{0.047} \\
\hline Ectasia & $9(45)$ & $3(15)$ & \\
\hline Small Aneurysm & $O(0)$ & $O(0)$ & \\
\hline Moderate Aneurysm & $1(5)$ & $1(5)$ & \\
\hline Giant Aneurysm & $O(0)$ & $O(0)$ & \\
\hline \multicolumn{3}{|c|}{ Third Echocardiography } & \multirow[t]{5}{*}{$\leq 0.001$} \\
\hline Ectasia & $O(0)$ & $O(0)$ & \\
\hline Small Aneurysm & $1(5)$ & $1(5)$ & \\
\hline Moderate Aneurysm & $O(0)$ & $0(0)$ & \\
\hline Giant Aneurysm & $O(0)$ & $0(0)$ & \\
\hline
\end{tabular}

After two weeks, the rate of improvement in coronary artery abnormalities was significantly different than before treatment in each group $(P=0.047)$. In the case group, 5 participants $(80 \%)$ and in the control group, 5 participants $(50 \%)$ showed coronary artery lesion improvement on the second echocardiography (Table 3).

After two months, in each group, only one patient had a small coronary artery aneurysm, and also, the rate of improvement in coronary artery abnormalities was significantly different compared to before treatment in each group $(\mathrm{P} \leq 0.001)$ (Table 3$)$.

The most involved coronary arteries in both groups were LMCA, RCA, and LAD, respectively, and there was no significant difference between the two groups. There were no other comorbidities, such as congenital heart disease and aortic valve insufficiency in any patient, and only tricuspid valve regurgitation was present in both groups in approximately the same proportion (Table 4). During the study, it was observed that none of the patients had significant side effects of the drug or showed adverse reactions to the drug. 
Table 4

Coronary artery involvement and other echocardiographic abnormality in Kawasaki disease patients

\begin{tabular}{|llll|}
\hline Coronary artery involvement & $\begin{array}{l}\text { IVIG group } \\
\text { Total number(\%) }\end{array}$ & $\begin{array}{l}\text { IVMP group } \\
\text { Total number(\%) }\end{array}$ & P. value \\
\hline RCA & $4(20)$ & $2(10)$ & 0.4 \\
\hline LMCA & $12(60)$ & $8(40)$ & 0.3 \\
\hline LAD & $3(15)$ & $3(15)$ & 0.9 \\
\hline Other abnormality & & & 0.6 \\
\hline Tricuspid regurgitation & $4(20)$ & $4(20)$ & \\
\hline IVIG: Intravenous Immunoglobulin; IVMP: Intra Venous Methylprednisolone Pulse \\
\hline
\end{tabular}

\section{Discussion}

Aspirin and IVIG are the recommended initial therapy in KD (12). As the pathology of KD consists of an immune-mediated panvasculitis of small and medium-sized muscular arteries, corticosteroids with their well-known and strong anti-inflammatory properties are expected to be of benefit. The available literature is contradictory $(13,14,15)$, even though the RAISE study has provided evidence for the advantage of corticosteroids plus intravenous immunoglobulin in high-risk patients with KD (16).

This may well be due to the different modes of using steroids in KD. Some use it as 'rescue therapy' (administered in children who fail initial IVIG therapy), whereas others address 'primary therapy' (corticosteroids administration as a component of first-line therapy, which may or may not include IVIG) (17). The differences between these studies could also have been due to the differing dose of aspirin (30 $\mathrm{mg} / \mathrm{kg}$ vs. $80-100 \mathrm{mg} / \mathrm{kg}$ ) or steroids (prednisolone $2 \mathrm{mg} / \mathrm{kg} /$ thrice-a-day vs. IV methylprednisolone 30 $\mathrm{mg} / \mathrm{Kg}$ once-a-day), or even IVIG (1 g/Kg/day for 2 days vs. $2 \mathrm{~g} / \mathrm{Kg}$ once). However, none of the previous studies has used the regimen, which we had to use due to pressing circumstances. Due to resource constraints, in this study, we could only use aspirin and corticosteroids, and follow-up showed significant coronary artery improvement and the presence of only one patient with a small-sized coronary aneurysm in each group.

Corticosteroid therapy has undoubtedly earned a place among the therapeutic regimens for KD. The link between the systemic immune response seen in the acute phase of KD and subsequent damage to the coronary arteries may be related to TNF- $a$ and its downstream effector molecules as the key players in mediating coronary artery damage. Several factors, such as prolonged fever and younger and older age, have been identified as part of the high-risk phenotype for poor coronary artery outcomes in KD (18). In this study, we included patients aged six months to five years, and the duration of fever and the acute 
phase of the disease was relatively similar between the two groups, so we had no additional factor in increasing the risk of coronary artery involvement.

In some previous studies, the use of steroids in the treatment of KD (first-line or refractory cases) has also been shown to reduce the duration of fever cessation and normalization of $\operatorname{CRP}(15,19,20,21)$.

Masaru et al. showed the changes in the inflammatory cytokine levels in the IVMP group compared with additional IVIG induced faster resolution of fever. The stronger suppression of MCP-1 and TNF-a levels by IVMP is noteworthy for preventing coronary artery lesions (10).

Corticosteroids have been considered contraindicated in KD because of a higher incidence of coronary aneurysms in the group treated with corticosteroids than in the untreated group (22). However, this finding can be criticized on several points, including drug dosage, being insufficient due to its oral administration, and the timing of the initiation of the steroid therapy being too late. As discussed above, anti-inflammatory agents are not used conventionally in sufficient amounts. Pulsed administration regimens have renewed interest in corticosteroids, particularly very high doses of $250-1000 \mathrm{mg}$ methylprednisolone (MP) for one to five days, as they are reported to be well-tolerated, more immunomodulatory than immunosuppressive, and safe, with only minor dose-related side effects (23, 24). The type I interferon pathway is affected in patients treated with pulse corticosteroids but not oral corticosteroids. Pulse corticosteroids markedly but transiently decreased the number of plasmacytoid dendritic cells (25).

In our study, the frequency of coronary artery involvement in the steroid pulse group in the two weeks after treatment was not significantly different from before treatment. As mentioned in the results section, patients in IVMP treated group, like patients receiving standard treatment with IVIG, had a marked improvement in coronary artery involvement and had the same percentage and type of involvement at the end of the study.

According to a study by Kijima et al., intravenous methylprednisolone pulse could significantly reduce coronary artery damage in children with KD (26). Shinohara et al. also showed a possible role of corticosteroids in treating the acute phase of KD (20). Swati Singhal et al. described the course of a oneyear-old child with KD who was treated with aspirin and corticosteroids as the initial therapy, and followup showed the presence of a small-sized coronary aneurysm (27).

The most compelling evidence for steroid use in KD comes from the meta-analysis done by Wooditch et al. (13). They performed a meta-analysis of 862 children and found a significant reduction in the incidence of coronary artery aneurysms among patients who received corticosteroids and aspirin with/without IVIG compared with aspirin alone or with IVIG. Newer studies are now testing steroid plus IVIG-aspirin combination in those not responding to the initial therapy or those likely to have a more resistant disease $(15,16,28,29)$. 
Based on the available evidence, this lack of efficacy of corticosteroids in first-line combination therapy in some patients may be due to reduced corticosteroid receptor gene expression on mRNA in these populations (15).

The basic idea of our pulse therapy was to prevent or suppress the process of aneurysm development by treating coronary angiitis, which may underlie coronary changes in KD, using large doses of corticosteroids parenterally within a relatively short period in the early stage of the disease. The present study indicates that steroid pulse therapy is effective in patients having some dilating changes of the coronary arteries and patients in whom an aneurysm formation is developing. Theoretically, such a treatment should be initiated before any dilative lesions appear in the acute phase of KD. In the acute phase, the activation of the innate immune system is demonstrated cytokine storm. In IVIG-resistance KD patients and patients with coronary artery involvements, TNFa, IL6 is higher than IVIG-Responder patients. $(30,31)$ Methylprednisolone pulses, especially in suppressing the innate immune system during the acute phase of $\mathrm{KD}$, may have a more pivotal role in inhibiting progressive coronary involvements than IVIG as the first-line treatment.

It should be emphasized that we found no significant side effects in any patient and that there was no convincing evidence that steroids made the coronary lesions worse.

Therefore, at present, intense corticosteroid pulse therapy for the coronary artery's inflammatory process may be used to prevent or minimize any tragic sequel of KD. Further evaluation with more sample size might be more valuable for IVIG and IVMP comparisons as the first-line treatment in KD, especially in anticipation of IVIG-resistance patients.

This is the first study on IVMP pulse therapy as first line treatment of KD, alone as a clinical trial study. However, Single blind study and 2 month follow-up are the main limitation of this study

\section{Conclusion}

Our study showed that intravenous methylprednisolone pulse also could significantly reduce coronary artery damage in children with KD. The price for IVIG in the Iranian setting is high, whereas Methylprednisolone is relatively less. If a definitive benefit or even equivalence between initial aspirin plus corticosteroid and the standard regimen can be conclusively demonstrated in large RCTs, the use of corticosteroids may be justified.

\section{Abbreviations}

KD: Kawasaki Disease

IVIG: Intravenous Immunoglobulin

IVMP: Intra Venous Methylprednisolone Pulse 
AHA: American Heart Association

RCA: Right Coronary Artery

LMCA: Left Main Coronary Artery

LCX: Left Circumflex

LAD: Left Anterior Descending

TNF-a: Tumor Necrosis Factor-a

MCP-1: monocyte chemoattractant protein-1

CBC: Cell Blood Count

ESR: Eerythrocyte Sedimentation Rate

CRP: C-reactive protein

SGOT: Serum Glutamic-Oxaloacetic Transaminase

SGPT: Serum Glutamic-Pyruvic Transaminase

\section{Declarations}

Acknowledgements: We would like to thank patients and their family for giving permission to publish their data.

Disclosures: None

Ethical Approval and Consent to participate: Written informed consent was gained from all patients or parents.

Trial registration: Our randomized, single-blind clinical trial, registered in Iranian Registry of Clinical Trials. (ClinicalTrials.IRCT Identifier: IRCT20181202041817N1) 26 Aug 2019, https://www.irct.ir/trial/35607

Consent for publication: Written informed consent was gained from patients or parents for publication.

Availability of supporting data: Requests for access to the data, which gathered and analysed during the current study are available from the corresponding author, V.Z.

Competing interests: The authors declare that they have no competing interests.

Funding: This work was funded and supported by Tehran University of Medical Sciences. (Grant ID: 9711465001) 


\section{Authors' contributions:}

N.A. performed data gathering and drafting of the manuscript

S.R., and F.T., contributed to the conception and design of the study, and clinical expertise

R.A., and P.S., analysed and interpreted the clinical data, and clinical expertise

E.A. performed echocardiography and contributed to interpretation of the clinical data

V.Z. provided the concept the survey, interpretation of the clinical data, oversaw the project and critical revision of the final draft of manuscript, primary responsibility for the paper.

All authors read and approved the final version of the manuscript.

\section{Authors' information:}

1. Children's Medical Center, Pediatrics Center of Excellence, Tehran, Iran

2. Department of Pediatrics, Isfahan University of Medical Sciences, Tehran, IR Iran

3. Department of Pediatrics, Tehran University of Medical Sciences, Tehran, IR Iran

4. Pediatric

5. Pediatric Rheumatology Research Group, Rheumatology Research Center, Tehran University of Medical Sciences, Tehran, Iran

\section{References}

1. Burns JC, Glode MP. Kawasaki syndrome. Lancet. 2004;364(9433):533-44.

2. Marrani E, Burns JC, Cimaz R. How should we classify Kawasaki disease. Front Immunol. 2018;9:2974.

3. Uehara R, Belay ED. Epidemiology of Kawasaki disease in Asia, Europe, and the United States. J Epidemiol. 2012;22(2):79-85.

4. Moradinejad M, Kiani A. Kawasaki Disease in 159 Iranian Children. Iran J Pediatr. 2007;17(3):241-6.

5. Research Committee on Kawasaki Disease. Report of Subcommittee on Standardization of Diagnostic Criteria and Reporting of Coronary Artery Lesions in Kawasaki Disease. Tokyo: Ministry of Health and Welfare; 1984.

6. McCrindle BW, Rowley AH, Newburger JW, et al. Diagnosis, treatment, and long-term management of Kawasaki disease: a scientific statement for health professionals from the American Heart Association. Circulation. 2017;135(17):e927-e99.

7. Furusho K, Kamiya T, Nakano H, et al. High-dose intravenous gammaglobulin for Kawasaki disease. Lancet. 1984;2(8411):1055-8. 
8. Barahona Afonso AF, Pires Joao CM. The Production Processes and Biological Effects of Intravenous Immunoglobulin. Biomolecules. 2016;6(1):15.

9. Li X, Chen Y, Tang Y, Ding Y, Xu Q, Sun L, Qian W, Qian G, Qin L, Lv H. Predictors of intravenous immunoglobulin-resistant Kawasaki disease in children: a meta-analysis of 4442 cases. Eur J Pediatr. 2018;177(8):1279-92.

10. Miura M, Kohno K, Ohki H, Yoshiba S. Effects of methylprednisolone pulse on cytokine levels in Kawasaki disease patients unresponsive to intravenous immunoglobulin. Eur J Pediatr. 2008;167:1119-23.

11. Chang L, Kuo H. The role of corticosteroids in the treatment of Kawasaki disease. Expert Rev Anti Infect Ther. 2020;18(2):155-64.

12. Moussa T, Wagner-Weiner L. Kawasaki Disease: Beyond IVIG and Aspirin. Pediatr Ann. 2019 Oct 1;48(10):e400-e405.

13. Wooditch AC, Aronoff SC. Effect of initial corticosteroid therapy on coronary artery aneurysm formation in Kawasaki disease: a meta-analysis of 862 children. Pediatrics. 2005;116:989-95.

14. Athappan G, Gale S, Ponniah T. Corticosteroid therapy for primary treatment of Kawasaki disease weight of evidence: a meta-analysis and systematic review of the literature. Cardiovasc J Afr. 2009;20:233-36.

15. Zhu BH, Lv HT, Sun L, et al. A meta-analysis on the effect of corticosteroid therapy in Kawasaki disease. Eur J Pediatr. 2012;171:571-78.

16. Kobayashi T, Saji T, Otani T, et al. Efficacy of immunoglobulin plus prednisolone for prevention of coronary artery abnormalities in severe Kawasaki disease (RAISE study): a randomised, open-label, blinded-endpoints trial. Lancet. 2012;379(9826):1613-20.

17. Shulman ST. Is there a role for corticosteroids in Kawasaki disease? J Pediatr. 2003;142:601-03.

18. Stringer E, Yeung RSM. Pathogenesis of Kawasaki disease: the central role of TNF-a. Future Rheumatol. 2008;3(1):69-77.

19. Newburger JW, Sleeper LA, McCrindle BW, et al. Randomized trial of pulsed corticosteroid therapy for primary treatment of Kawasaki disease. NEJM. 2007;356(7):663-75.

20. Shinohara M, Sone K, Tomomasa T, Morikawa A. Corticosteroids in the treatment of the acute phase of Kawasaki disease. J Pediatr. 1999;135(4):465-9.

21. Sundel R. Clarifying the role of corticosteroids in Kawasaki disease. JAMA Pediatr. 2016;170(12):1140-2.

22. Kato H, Koike S, Yokoyama T. Kawasaki disease: Effect of treatment on coronary involvement. Pediatrics. 1979;63:175.

23. Parker BJ, Bruce IN. High dose methylprednisolone therapy for the treatment of severe systemic lupus erythematosus. Lupus. 2007;16(6):387-93.

24. Franchin G, Diamond B. Pulse steroids: how much is enough? Autoimmun Rev. 2006;5(2):111-3. 
25. Guiducci C, Gong M, Xu Z, et al. TLR recognition of self nucleic acids hampers glucocorticoid activity in lupus. Nature. 2010;465(7300):937-41.

26. Kijima Y, Kamiya T, Suzuki A, Hirose O, Manabe H. A trial procedure to prevent aneurysm formation of the coronary arteries by steroid pulse therapy in Kawasaki disease. Jpn Circ J. 1982;46(11):1239-42.

27. Singhal S, Mishra D, Kurien S, Juneja M. Primary treatment of kawasaki disease with corticosteroids. J Clin Diag Res. 2014;8(5):PD04-5.

28. Ogata S, Ogihara $Y$, Honda $T$, et al. Corticosteroid pulse combination therapy for refractory Kawasaki disease: A randomized trial. Pediatrics. 2012;129:e17.

29. Wardle AJ, Connolly GM, Seager MJ, Tulloh RM. Corticosteroids for the treatment of Kawasaki disease in children. Cochrane Database Syst Rev. 2017;1(1):CD011188.

30. Matsubara T, Ichiyama T, Furukawa S. Immunological profile of peripheral blood lymphocytes and monocytes/macrophages in Kawasaki disease. Clin Exp Immunol. 2005;141(3):381-7.

31. Hur G, Song MS, Sohn S, et al. Infliximab treatment for intravenous immunoglobulin-resistant Kawasaki disease: A multicenter study in Korea. Korean Circ J. 2019;49(2):183-91.

\section{Figures}




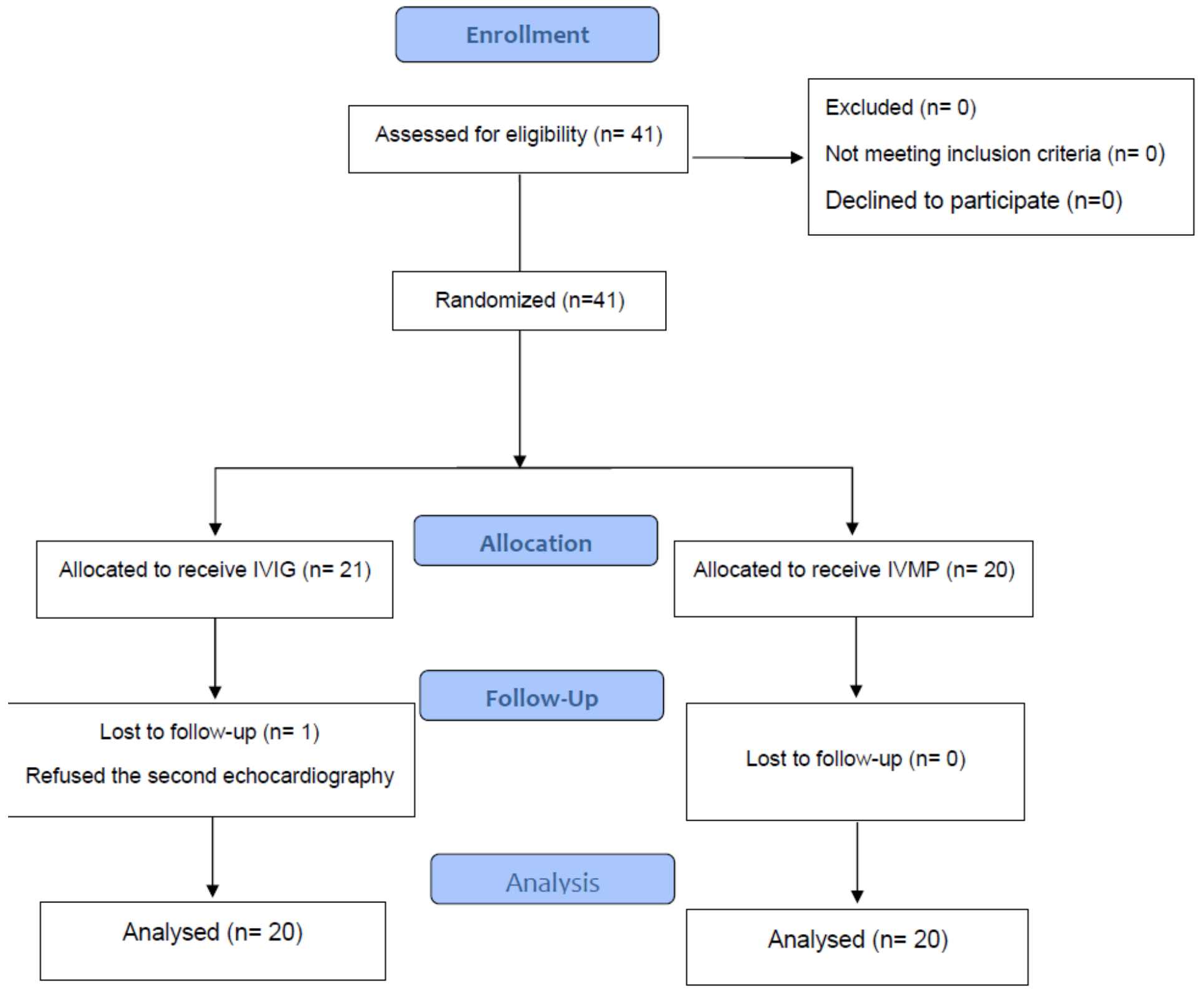

Figure 1

CONSORT flow chart of the randomized clinical trial with intravenous methylprednisolone pulse administration in children with KD 